\title{
Stability and Bifurcation Analysis for a Class of Generalized Reaction-Diffusion Neural Networks with Time Delay
}

\author{
Tianshi Lv, Qintao Gan, and Qikai Zhu \\ Institute of Applied Mathematics, Shijiazhuang Mechanical Engineering College, Shijiazhuang 050003, China \\ Correspondence should be addressed to Qintao Gan; ganqintao@sina.com
}

Received 5 July 2015; Revised 3 August 2015; Accepted 10 August 2015

Academic Editor: Carlo Bianca

Copyright (C) 2016 Tianshi Lv et al. This is an open access article distributed under the Creative Commons Attribution License, which permits unrestricted use, distribution, and reproduction in any medium, provided the original work is properly cited.

\begin{abstract}
Considering the fact that results for static neural networks are much more scare than results for local field neural networks and our purpose letting the problem researched be more general in many aspects, in this paper, a generalized neural networks model which includes reaction-diffusion local field neural networks and reaction-diffusion static neural networks is built and the stability and bifurcation problems for it are investigated under Neumann boundary conditions. First, by discussing the corresponding characteristic equations, the local stability of the trivial uniform steady state is discussed and the existence of Hopf bifurcations is shown. By using the normal form theory and the center manifold reduction of partial function differential equations, explicit formulae which determine the direction and stability of bifurcating periodic solutions are acquired. Finally, numerical simulations show the results.
\end{abstract}

\section{Introduction}

In the past several decades, the dynamics of neural networks have been extensively investigated.

The artificial neural network has been used widely in various fields such as signal processing, pattern recognition, optimization, associative memories, automatic control engineering, artificial intelligence, and fault diagnosis, because it has the characteristics of self-adaption, self-organization, and self-learning.

Most of the phenomena occurring in real-world complex systems do not have an immediate effect but appear with some delay; for example, there exist time delays in the information processing of neurons. Therefore, time delays have been inserted into mathematical models and in particular in models of the applied sciences based on ordinary differential equations. The delayed axonal signal transmissions in the neural network models make the dynamical behaviors become more complicated, because a time delay into an ordinary differential equation could change the stability of the equilibrium (stable equilibrium becomes unstable) and could cause fluctuations, and Hopf bifurcation can occur (see [1]). And in [1] we can know the time delays' effects from the work by Carlo Bianca, Massimiliano Ferrara, and Luca Guerrini. So, the delay is an important control parameter.

In addition, we must consider that the activations vary in space as well as in time, because the electrons move in asymmetric electromagnetic fields, and there exists diffusion in neural network (see [2]).

In the past, the main work was to research local field neural networks, and static neural networks were rarely studied. Considering the fact that the problem of generalized neural network is more general in many aspects; in this paper, we will investigate a class of generalized neural networks which combine local field neural networks and static neural networks.

In order to study the effect of time delays and diffusion on the dynamics of a neural network model, in [3], Gan and Xu considered the following neural network model:

$$
\begin{aligned}
& \frac{\partial u_{1}}{\partial t}=D_{1} \Delta u_{1}-a_{1} u_{1}(t, x)+g_{1}\left(u_{2}(t-\tau, x)\right), \\
& \frac{\partial u_{2}}{\partial t}=D_{2} \Delta u_{2}-a_{2} u_{2}(t, x)+g_{2}\left(u_{1}(t-\tau, x)\right) .
\end{aligned}
$$


Motivated by the works of Gan and $\mathrm{Xu}$, in this paper, we are concerned with the following neural network system with time delay and reaction-diffusion:

$$
\begin{aligned}
\frac{\partial u}{\partial t}= & D_{1} \Delta u-a_{1} u(t, x)+b_{11} g_{1}\left(w_{11} u(t, x)\right) \\
& +b_{12} g_{2}\left(w_{12} v(t-\tau, x)\right), \quad t>0, x \in \Omega, \\
\frac{\partial v}{\partial t}= & D_{2} \Delta v-a_{2} v(t, x)+b_{21} g_{1}\left(w_{21} u(t-\tau, x)\right) \\
& +b_{22} g_{2}\left(w_{22} v(t, x)\right), \quad t>0, x \in \Omega
\end{aligned}
$$

with initial and boundary conditions (Neumann boundary conditions):

$$
\begin{aligned}
& \frac{\partial u}{\partial l}=\frac{\partial v}{\partial l}=0, \quad t>0, x \in \partial \Omega, \\
& u(t, x)=\varphi_{1}(t, x), \\
& v(t, x)=\varphi_{2}(t, x), \\
& t \in[-\tau, 0], x \in \bar{\Omega},
\end{aligned}
$$

where $a_{1}, a_{2}, \tau, D_{1}, D_{2} \geq 0, b_{11}, b_{12}, b_{21}, b_{22}, w_{11}, w_{12}, w_{21}$, and $w_{22}$ are random constants, where $a_{1}$ and $a_{2}$ represent the neuron charging time constants, $\tau$ represents the signal transmission time delay, $D_{1}$ and $D_{2}$ represent the smooth diffusion operators, $b_{11}, b_{12}, b_{21}$, and $b_{22}$ represent connecting weight coefficients, and $w_{11}, w_{12}, w_{21}$, and $w_{22}$ represent the coefficients of $u(t, x), v(t-\tau, x), u(t-\tau, x)$, and $v(t, x)$, respectively. $u, v$, and $x$ are the state variables and space variable, respectively. $g_{1}$ and $g_{2}$ are the action functions of the neurons satisfying $g_{1}(0)=g_{2}(0)=0 . \Omega$ is a bounded domain in $R^{n}$ with smooth boundary $\partial \Omega$, where $\partial / \partial l$ denotes the outward normal derivative on $\partial \Omega$.

The organization of this paper is as follows. In Section 2, by analyzing the corresponding characteristic equations, we discuss the local stability of trivial uniform steady state and the existence of Hopf bifurcations of (2) and (3). In Section 3, by applying the normal form and the center manifold theorem, closed-form expressions are derived which allow us to determine the direction of the Hopf bifurcations and the stability of the periodic solutions in (2) and (3) (see [2]). In Section 4, numerical simulations are carried out to illustrate the main theoretical results.

\section{Local Stability and Hopf Bifurcation}

Obviously, we can easily show that system (2) always has a trivial uniform steady state $E^{*}=(0,0)$.

Here, we use $0=\mu_{1}<\mu_{2}<\cdots$ as the eigenvalues of the operator $-\Delta$ on $\Omega$ with the homogeneous Neumann boundary conditions and $E\left(\mu_{i}\right)$ as the eigenspace corresponding to $\mu_{i}$ in $C^{1}(\Omega)$. Let $\nabla=\left[C^{1}(\Omega)\right]^{2}$, let $\left\{\varphi_{i j} ; j=1, \ldots, \operatorname{dim} E\left(\mu_{i}\right)\right\}$ be an orthonormal basis of $E\left(\mu_{i}\right)$, and let $\nabla_{i j}=\left\{c \varphi_{i j} \mid c \in R^{2}\right\}$. Then,

$$
\begin{aligned}
& \nabla=\bigoplus_{i=1}^{\infty} \nabla_{i}, \\
& \nabla_{i}=\bigoplus_{j=1}^{\operatorname{dim} E\left(\mu_{i}\right)} \nabla_{i j} .
\end{aligned}
$$

Let $\wp=\operatorname{diag}\left(D_{1}, D_{2}\right), \zeta m=\wp \Delta m+\mathbb{Z}\left(E^{*}\right) m$, where

$$
\begin{aligned}
& \mathbb{Z}\left(E^{*}\right) m \\
& =\left(\begin{array}{cc}
-a_{1}+b_{11} w_{11} g_{1}^{\prime}(0) & 0 \\
0 & -a_{2}+b_{22} w_{22} g_{2}^{\prime}(0)
\end{array}\right)\left(\begin{array}{l}
u(t, x) \\
v(t, x)
\end{array}\right) \\
& \quad+\left(\begin{array}{cc}
0 & b_{12} w_{12} g_{2}^{\prime}(0) \\
b_{21} w_{21} g_{1}^{\prime}(0) & 0
\end{array}\right)\left(\begin{array}{l}
u(t-\tau, x) \\
v(t-\tau, x)
\end{array}\right) .
\end{aligned}
$$

First, we linearize system (2) at $E^{*}$. Then, $m_{t}=\zeta m . \nabla_{i}$ is invariant under the operator $\zeta$ for each $i \geq 1$, and $\lambda$ is an eigenvalue of $\zeta$ if and only if it is an eigenvalue of the matrix $-\mu_{i} \wp+\mathbb{Z}\left(E^{*}\right)$ for some $i \geq 1$, in which case, there is an eigenvalue in $\nabla_{i}$.

The characteristic equation of $-\mu_{i} \wp+\mathbb{Z}\left(E^{*}\right)$ is of the form

$$
\lambda^{2}+p_{1} \lambda+p_{2}+p_{3} e^{-2 \lambda \tau}=0
$$

where

$$
\begin{aligned}
p_{1} & =\mu_{i} D_{1}+a_{1}-b_{11} w_{11} g_{1}^{\prime}(0)+\mu_{i} D_{2}+a_{2} \\
& -b_{22} w_{22} g_{2}^{\prime}(0), \\
p_{2} & =\left(\mu_{i} D_{1}+a_{1}-b_{11} w_{11} g_{1}^{\prime}(0)\right) \\
& \cdot\left(\mu_{i} D_{2}+a_{2}-b_{22} w_{22} g_{2}^{\prime}(0)\right), \\
p_{3} & =-b_{12} b_{21} w_{12} w_{21} g_{1}^{\prime}(0) g_{2}^{\prime}(0) .
\end{aligned}
$$

Letting $\tau=0$, then (6) becomes

$$
\lambda^{2}+p_{1} \lambda+p_{2}+p_{3}=0 .
$$

Obviously,

$$
\begin{aligned}
p_{2}+p_{3}= & \left(\mu_{i} D_{1}+a_{1}-b_{11} w_{11} g_{1}^{\prime}(0)\right) \\
& \cdot\left(\mu_{i} D_{2}+a_{2}-b_{22} w_{22} g_{2}^{\prime}(0)\right) \\
& -b_{12} b_{21} w_{12} w_{21} g_{1}^{\prime}(0) g_{2}^{\prime}(0) .
\end{aligned}
$$

Obviously, if the following holds:

(H1)

$$
\begin{aligned}
& a_{1}-b_{11} w_{11} g_{1}^{\prime}(0)>0, \\
& a_{2}-b_{22} w_{22} g_{2}^{\prime}(0)>0, \\
& \left(a_{1}-b_{11} w_{11} g_{1}^{\prime}(0)\right)\left(a_{2}-b_{22} w_{22} g_{2}^{\prime}(0)\right) \\
& \quad-b_{12} b_{21} w_{12} w_{21} g_{1}^{\prime}(0) g_{2}^{\prime}(0)>0
\end{aligned}
$$


then $p_{2}+p_{3}>0, p_{1}>0$. Hence, if (H1) holds, when $\tau=0$, the trivial uniform steady state $E^{*}$ of problems (2) and (3) is locally stable.

Let $i \omega(\omega>0)$ be a solution of (6), separating real and imaginary parts; then, we can get that

$$
\begin{aligned}
\omega^{2}-p_{2} & =p_{3} \cos 2 \omega \tau, \\
p_{1} \omega & =p_{3} \sin 2 \omega \tau .
\end{aligned}
$$
that

Squaring and adding the two equations of (11), we obtain

$$
\omega^{4}+\left(p_{1}^{2}-2 p_{2}\right) \omega^{2}+p_{2}^{2}-p_{3}^{2}=0
$$

Letting $z=\omega^{2}$, then (12) becomes

$$
z^{2}+\left(p_{1}^{2}-2 p_{2}\right) z+p_{2}^{2}-p_{3}^{2}=0
$$

Obviously, it is easy to calculate that

$$
\begin{gathered}
p_{1}^{2}-2 p_{2}=\left(\mu_{i} D_{1}+a_{1}-b_{11} w_{11} g_{1}^{\prime}(0)\right)^{2} \\
+\left(\mu_{i} D_{2}+a_{2}-b_{22} w_{22} g_{2}^{\prime}(0)\right)^{2}>0 \\
p_{2}^{2}-p_{3}^{2}=\left(\mu_{i} D_{1}+a_{1}-b_{11} w_{11} g_{1}^{\prime}(0)\right)^{2} \\
\cdot\left(\mu_{i} D_{2}+a_{2}-b_{22} w_{22} g_{2}^{\prime}(0)\right)^{2} \\
-b_{12}^{2} b_{21}^{2} w_{12}^{2} w_{21}^{2} g_{1}^{\prime 2}(0) g_{2}^{\prime 2}(0) .
\end{gathered}
$$

Let

$$
\begin{aligned}
q_{1}= & \left(a_{1}-b_{11} w_{11} g_{1}^{\prime}(0)\right)\left(a_{2}-b_{22} w_{22} g_{2}^{\prime}(0)\right) \\
& +b_{12} b_{21} w_{12} w_{21} g_{1}^{\prime}(0) g_{2}^{\prime}(0), \\
q_{2}= & \left(a_{1}-b_{11} w_{11} g_{1}^{\prime}(0)\right)^{2}\left(a_{2}-b_{22} w_{22} g_{2}^{\prime}(0)\right)^{2} \\
& -b_{12}^{2} b_{21}^{2} w_{12}^{2} w_{21}^{2} g_{1}^{\prime 2}(0) g_{2}^{\prime 2}(0), \\
q_{3}= & \left(a_{1}-b_{11} w_{11} g_{1}^{\prime}(0)\right)^{2}+\left(a_{2}-b_{22} w_{22} g_{2}^{\prime}(0)\right)^{2}>0 .
\end{aligned}
$$

Therefore, if $q_{2}>0$, (13) has no positive roots. Then, if $q_{1}>0$ and $(H 1)$ Holds, the trivial uniform steady state $E^{*}$ of system (2) is locally asymptotically stable for all $i \geq 1$ and $\tau \geq 0$.

For $i=1$, if $q_{1}<0$, then (12) has a unique positive root $\omega_{0}$, where

$$
\omega_{0}=\left(\frac{1}{2}\left(-q_{3}+\sqrt{q_{3}^{2}-4 q_{2}}\right)\right)^{1 / 2} .
$$

It means that the characteristic equation (6) admits a pair of purely imaginary roots of the form $\pm i \omega_{0}$ for $i=1$.

Take $\omega=\left((1 / 2)\left(-q_{3}+\sqrt{q_{3}^{2}-4 q_{2}}\right)\right)^{1 / 2}$. Obviously, (12) holds if and only if $i=1$. Now, we define that

$$
\tau_{0 n}=\frac{1}{2 \omega_{0}} \arccos \frac{\omega_{0}^{2}-p_{2}}{p_{3}}+\frac{n \pi}{\omega_{0}}, \quad n=0,1, \ldots
$$

Then, for $i=1$, when $\tau=\tau_{0 n}$, (6) has a pair of purely imaginary roots $\pm i \omega_{0}$ and all roots of it have negative real parts for $i \geq 2$. It is easy to see that if $(H 1)$ holds, the trivial uniform steady state $E^{*}$ is locally stable for $\tau=0$. Hence, on the basis of the general theory on characteristic equations of delay-differential equations from [3, Theorem 4.1], we can know that $E^{*}$ remains stable when $\tau<\tau_{0}$, where $\tau_{0}=\tau_{00}$.

Now, we claim that

$$
\left.\frac{d(\operatorname{Re} \lambda)}{d \tau}\right|_{\tau=\tau_{0}}>0
$$

This will mean that there exists at least one eigenvalue with positive real part when $\tau>\tau_{0}$. In addition, the conditions for the existence of a Hopf bifurcation [2] are then satisfied generating a periodic solution. To this end, we differentiate (6) about $\tau$; then,

$$
\left(2 \lambda+p_{1}\right) \frac{d \lambda}{d \tau}-2 p_{3} e^{-2 \lambda \tau}\left(\lambda+\tau \frac{d \lambda}{d \tau}\right)=0 .
$$

So, we know that

$$
\begin{aligned}
\left(\frac{d \lambda}{d \tau}\right)^{-1} & =\frac{2 \lambda+p_{1}-2 \tau p_{3} e^{-2 \lambda \tau}}{2 \lambda p_{3} e^{-2 \lambda \tau}} \\
& =\frac{\left(2 \lambda+p_{1}\right) e^{2 \lambda \tau}}{2 \lambda p_{3}}-\frac{\tau}{\lambda}
\end{aligned}
$$

Therefore,

$$
\begin{aligned}
& \operatorname{sign}\left\{\frac{d(\operatorname{Re} \lambda)}{d \tau}\right\}_{\lambda=i \omega_{0}}=\operatorname{sign}\left\{\operatorname{Re}\left(\frac{d \lambda}{d \tau}\right)^{-1}\right\}_{\lambda=i \omega_{0}} \\
& =\operatorname{sign}\left\{\operatorname{Re}\left[\frac{\left(2 \lambda+p_{1}\right) e^{2 \lambda \tau}}{2 \lambda p_{3}}\right]_{\lambda=i \omega_{0}}+\operatorname{Re}\left[-\frac{\tau}{\lambda}\right]_{\lambda=i \omega_{0}}\right\} \\
& =\operatorname{sign}\left\{\frac{2 \omega_{0} \cos 2 \omega_{0} \tau+p_{1} \sin 2 \omega_{0} \tau}{2 \omega_{0} p_{3}}\right\} .
\end{aligned}
$$

By (11), we can obtain that

$$
\operatorname{sign}\left\{\frac{d(\operatorname{Re} \lambda)}{d \tau}\right\}_{\lambda=i \omega_{0}}=\operatorname{sign}\left\{\frac{2 \omega_{0}^{2}+p_{1}^{2}-2 p_{2}}{2 p_{3}^{2}}\right\} .
$$

Because $p_{1}^{2}-2 p_{2}>0$, so

$$
\left.\frac{d(\operatorname{Re} \lambda)}{d \tau}\right|_{\tau=\tau_{0}, \omega=\omega_{0}}>0 .
$$

Hence, the transversal condition holds and a Hopf bifurcation occurs when $\omega=\omega_{0}$ and $\tau=\tau_{0}$.

Consequently, we gain the following results.

Theorem 1. Let $\tau_{0}=\tau_{00}$ and let $q_{1}$ be defined by (15). For system (2), let (H1) hold. If $q_{1}>0$, the trivial uniform steady state $E^{*}$ of system (2) is locally asymptotically stable when $\tau \geq$ 0 ; if $q_{1}<0$, the trivial uniform steady state $E^{*}$ is asymptotically stable for $0 \leq \tau<\tau_{0}$ and is unstable for $\tau>\tau_{0}$; furthermore, system (2) undergoes a Hopf bifurcation at $E^{*}$ when $\tau=\tau_{0}$. 


\section{Direction and Stability of Hopf Bifurcation}

In Section 2, we have demonstrated that systems (2) and (3) undergo a train of periodic solutions bifurcating from the trivial uniform steady state $E^{*}$ at the critical value of $\tau$. In this section, we derive explicit formulae to determine the properties of the Hopf bifurcation at critical value $\tau_{0}$ by using the normal form theory and center manifold reduction for PFDEs. In this section, we also let the condition $(H 1)$ hold and $q_{1}<0$. And the work of Bianca and Guerrini in papers [4-7] is the founder of the method in this section.
Set $\tau=\alpha+\tau_{0}$. We first should normalize the delay $\tau$ by the time-scaling $t \rightarrow t / \tau$. Then, (2) can be rewritten in the fixed phase space $\ell^{*}=C([-1,0], X)$ as

$$
\begin{aligned}
\dot{m}(t)= & \tau_{0} \wp \Delta m(t)+\tau_{0} \mathbb{Z}\left(E^{*}\right)(m(t)) \\
& +f^{*}(m(t), \alpha),
\end{aligned}
$$

where $f^{*}: \ell^{*} \times R^{+} \rightarrow R^{2}$ is defined by

$$
f^{*}(\phi, \alpha)=\alpha \wp \Delta \phi(0)+\tau_{0} \mathbb{Z}\left(E^{*}\right)(\phi)+\left(\tau_{0}+\alpha\right)\left(\begin{array}{c}
\frac{1}{2 !} b_{12} w_{12}^{2} g_{2}^{\prime \prime}(0) \phi_{2}^{2}(-1)+\frac{1}{3 !} b_{12} w_{12}^{3} g_{2}^{\prime \prime \prime}(0) \phi_{2}^{3}(-1)+\cdots \\
\frac{1}{2 !} b_{21} w_{21}^{2} g_{1}^{\prime \prime}(0) \phi_{1}^{2}(-1)+\frac{1}{3 !} b_{21} w_{21}^{3} g_{1}^{\prime \prime \prime}(0) \phi_{1}^{3}(-1)+\cdots
\end{array}\right)
$$

where $\phi=\left(\phi_{1}, \phi_{2}\right)^{T} \in \ell^{*}$.

By the discussion in Section 2, we can know that the origin $(0,0)$ is a steady state of $(24)$ and $\Lambda_{0}=\left\{-i \omega_{0} \tau_{0}, i \omega_{0} \tau_{0}\right\}$ are a pair of simple purely imaginary eigenvalues of the linear equation

$$
\dot{m}(t)=\tau_{0} \wp \Delta m(t)+\tau_{0} \mathbb{Z}\left(E^{*}\right)(m(t))
$$

and the functional differential equation

$$
\dot{z}(t)=\tau_{0} \mathbb{Z}\left(z_{t}\right) .
$$

On the basis of the Riesz representation theorem, there exists a function $\eta(\theta, \tau)$ of bounded variation for $\theta \in[-1,0]$ such that

$$
\mathbb{Z}\left(E^{*}\right)(\varphi)=\frac{1}{\tau_{0}} \int_{-1}^{0} d \eta\left(\theta, \tau_{0}\right) \varphi(\theta), \quad \text { where } \varphi \in \mathrm{C}
$$

Here, we choose that

$$
\begin{aligned}
& \eta\left(\theta, \tau_{0}\right) \\
= & \tau_{0}\left(\begin{array}{cc}
-a_{1}+b_{11} w_{11} g_{1}^{\prime}(0) & 0 \\
0 &
\end{array}\right) \delta(\theta) \\
& -\tau_{0}\left(\begin{array}{cc}
0 & b_{12} w_{12} g_{2}^{\prime}(0) \\
b_{21} w_{21} g_{1}^{\prime}(0) & 0
\end{array}\right) \delta(\theta+1),
\end{aligned}
$$

where $\delta$ is the Dirac delta function.

Let $A\left(\tau_{0}\right)$ denote the infinitesimal generator of the semigroup induced by the solutions of (27) and let $A^{*}$ be the formal adjoint of $A\left(\tau_{0}\right)$ under the bilinear pairing

$$
\begin{aligned}
\langle\psi(s), \varphi(\theta)\rangle= & \psi(0) \varphi(0) \\
& -\int_{-1}^{0} \int_{\xi=0}^{\theta} \psi(\xi-\theta) d \eta(\theta) \varphi(\xi) d \xi,
\end{aligned}
$$

where $\varphi \in C^{1}\left([-1,0], R^{2}\right), \psi \in C^{1}\left([0,1],\left(R^{2}\right)^{*}\right), \eta(\theta)=$ $\eta\left(\theta, \tau_{0}\right)$. Then, $A\left(\tau_{0}\right)$ and $A^{*}$ are a pair of adjoint operators.

By the discussions in Section 2, we can realize that $A\left(\tau_{0}\right)$ has a pair of simple purely imaginary eigenvalues $\pm i w_{0} \tau_{0}$ and they are also eigenvalues of $A^{*}$ since $A\left(\tau_{0}\right)$ and $A^{*}$ are adjoint operators. Let $P$ and $P^{*}$ be the center spaces of $A\left(\tau_{0}\right)$ and $A^{*}$ associated with $\Lambda_{0}$, respectively. Hence, $P^{*}$ is the adjoint space of $P$ and $\operatorname{dim} P=\operatorname{dim} P^{*}=2$.

Let

$$
\begin{aligned}
& \gamma=\frac{b_{21} w_{21} g_{1}^{\prime}(0) e^{-i w_{0} \tau_{0}}}{a_{2}+i w_{0}-b_{22} w_{22} g_{2}^{\prime}(0)}, \\
& \kappa=\frac{b_{12} w_{12} g_{2}^{\prime}(0) e^{i w_{0} \tau_{0}}}{a_{2}-i w_{0}-b_{22} w_{22} g_{2}^{\prime}(0)}
\end{aligned}
$$

then,

$$
\begin{aligned}
& p_{1}(\theta)=e^{i w_{0} \tau_{0} \theta}(1, \gamma)^{T}, \\
& p_{2}(\theta)=\bar{p}_{1}(\theta),
\end{aligned}
$$

$$
-1 \leq \theta \leq 0
$$

is a basis of $P$ associated with $\Lambda_{0}$ and

$$
\begin{aligned}
& q_{1}(s)=(1, \kappa)^{T} e^{-i w_{0} \tau_{0} s}, \\
& q_{2}(s)=\bar{q}_{1}(s),
\end{aligned}
$$

$$
0 \leq s \leq 1
$$

is a basis of $Q$ associated with $\Lambda_{0}$.

Let $\Phi=\left(\Phi_{1}, \Phi_{2}\right)$, where

$$
\begin{aligned}
& \Phi_{1}(\theta)=\frac{p_{1}(\theta)+p_{2}(\theta)}{2}, \\
& \Phi_{2}(\theta)=\frac{p_{1}(\theta)-p_{2}(\theta)}{2 i}
\end{aligned}
$$


for $\theta \in[-1,0]$, and let $\Psi^{*}=\left(\Psi_{1}^{*}, \Psi_{2}^{*}\right)^{T}$, where

$$
\begin{aligned}
& \Psi_{1}^{*}(s)=\frac{q_{1}(s)+q_{2}(s)}{2}, \\
& \Psi_{2}^{*}(s)=\frac{q_{1}(s)-q_{2}(s)}{2 i}
\end{aligned}
$$

for $s \in[-1,0]$.

Now we define that $\left(\Psi^{*}, \Phi\right)=\left(\Psi_{j}^{*}, \Phi_{k}\right),(j, k=1,2)$, and construct a new basis $\Psi$ for $Q$ by

$$
\Psi=\left(\Psi_{1}^{*}, \Psi_{2}\right)^{T}=\left(\Psi^{*}, \Phi\right)^{-1} \Psi^{*} .
$$

Hence, $(\Psi, \Phi)=I_{2}$, which is the second-order identity matrix. Moreover, we define $f_{0}$ for $f_{0}=\left(\beta_{0}^{1}, \beta_{0}^{2}\right)$ and $c \cdot f_{0}=$ $c_{0} \beta_{0}^{1}+c_{2} \beta_{0}^{2}$ for $c=\left(c_{1}, c_{2}\right)^{T} \in C$. Then, the center space of linear equation (26) is given by $P_{C N} \ell^{*}$, where

$$
P_{C N} \ell^{*}=\Phi\left(\Psi,\left\langle\phi, f_{0}\right\rangle\right) \cdot f_{0}, \quad \phi \in \ell^{*},
$$

and $\ell^{*}$ denotes the complementary subspace of $P_{C N} \ell^{*}$, where

$$
\ell^{*}=P_{C N} \ell^{*} \oplus Q \text {. }
$$

Let $A_{\tau_{0}}$ be defined by

$$
\begin{aligned}
A_{\tau_{0}} \phi(\theta) & \\
= & \dot{\phi}(\theta) \\
& +X_{0}(\theta)\left[\wp \Delta \phi(0)+\tau_{0} \mathbb{Z}\left(E^{*}\right)(\phi(\theta))-\dot{\phi}(0)\right], \\
& \phi \in \ell^{*},
\end{aligned}
$$

where $X_{0}:[-1,0] \rightarrow B(X, X)$ is given by

$$
X_{0}(\theta)= \begin{cases}0, & -1 \leq \theta<0, \\ I, & \theta=0 .\end{cases}
$$

Then, we have rewritten system (24), and it can be rewritten as follows:

$$
\dot{m}(t)=A_{\tau_{0}} m(t)+X_{0} f^{*}(m(t), \alpha) .
$$

The solution of (24) on the center manifold is given by

$$
m^{*}(t)=\Phi\left(x_{1}, x_{2}\right)^{T} \cdot f_{0}+W\left(x_{1}, x_{2}, \alpha\right) \text {. }
$$

Letting $z=x_{1}-i x_{2}, W=W_{20}\left(z^{2} / 2\right)+W_{11} z \bar{z}+W_{02}\left(\bar{z}^{2} / 2\right)+$ $\cdots$, then

$$
\dot{z}=i w_{0} \tau_{0} z+g(z, \bar{z})
$$

where

$$
\begin{aligned}
g(z, \bar{z}) & =\left(\Psi_{1}(0)-i \Psi_{2}(0)\right)\left\langle f^{*}\left(m^{*}(t), 0\right), f_{0}\right\rangle \\
& \triangleq g_{20} \frac{z^{2}}{2}+g_{11} z \bar{z}+g_{02} \frac{\bar{z}^{2}}{2}+g_{21} \frac{z^{2} \bar{z}}{2}+\cdots .
\end{aligned}
$$

We can use some easy computations to show that

$$
\begin{aligned}
\left\langle f^{*}\left(m^{*}(t), 0\right), f_{0}\right\rangle= & \frac{\tau_{0}}{8}\left(\begin{array}{c}
c_{11} z^{2}+c_{12} \bar{z}^{2}+c_{13} z \bar{z} \\
c_{21} z^{2}+c_{22} \bar{z}^{2}+c_{23} z \bar{z}
\end{array}\right) \\
& +\frac{\tau_{0}}{16}\left(\begin{array}{c}
\left\langle c_{01}, 1\right\rangle \\
\left\langle c_{02}, 1\right\rangle
\end{array}\right) z^{2} \bar{z}+\cdots,
\end{aligned}
$$

where

$$
\begin{aligned}
& c_{11}=b_{12} w_{12} g_{2}^{\prime}(0)\left(-a_{1}+b_{11} w_{11} g_{1}^{\prime}(0)\right) \gamma e^{-i w_{0} \tau_{0}}, \\
& c_{12}=b_{12} w_{12} g_{2}^{\prime}(0)\left(-a_{1}+b_{11} w_{11} g_{1}^{\prime}(0)\right) \bar{\gamma} e^{i w_{0} \tau_{0}} \text {, } \\
& c_{13}=b_{12} w_{12} g_{2}^{\prime}(0)\left(-a_{1}+b_{11} w_{11} g_{1}^{\prime}(0)\right) \\
& \cdot\left(\bar{\gamma} e^{i w_{0} \tau_{0}}+\gamma e^{-i w_{0} \tau_{0}}\right) \\
& c_{21}=b_{21} w_{21} g_{1}^{\prime}(0)\left(-a_{2}+b_{22} w_{22} g_{1}^{\prime}(0)\right) \gamma e^{-i w_{0} \tau_{0}} \text {, } \\
& c_{22}=b_{21} w_{21} g_{1}^{\prime}(0)\left(-a_{2}+b_{22} w_{22} g_{2}^{\prime}(0)\right) \bar{\gamma} e^{i w_{0} \tau_{0}} \text {, } \\
& c_{23}=b_{21} w_{21} g_{1}^{\prime}(0)\left(-a_{2}+b_{22} w_{22} g_{2}^{\prime}(0)\right) \\
& \cdot\left(\gamma e^{i w_{0} \tau_{0}}+\bar{\gamma} e^{-i w_{0} \tau_{0}}\right) \\
& c_{01}=b_{12} w_{12} g_{2}^{\prime}(0)\left(-a_{1}+b_{11} w_{11} g_{1}^{\prime}(0)\right) \\
& \cdot\left(\bar{\gamma} W_{20}^{(1)}(0) e^{i w_{0} \tau_{0}}+W_{20}^{(2)}(-1)\right)+2 b_{12} w_{12} g_{2}^{\prime}(0) \\
& \cdot\left(-a_{1}+b_{11} w_{11} g_{1}^{\prime}(0)\right) \\
& \cdot\left(W_{11}^{(2)}(-1)+\gamma W_{11}^{(1)}(0) e^{-i w_{0} \tau_{0}}\right) \text {, } \\
& c_{02}=b_{21} w_{21} g_{1}^{\prime}(0)\left(-a_{2}+b_{22} w_{22} g_{2}^{\prime}(0)\right) \\
& \cdot\left(\bar{\gamma} W_{20}^{(1)}(-1)+W_{20}^{(2)}(0) e^{i w_{0} \tau_{0}}\right)+2 b_{21} w_{21} g_{1}^{\prime}(0) \\
& \cdot\left(-a_{2}+b_{22} w_{22} g_{2}^{\prime}(0)\right) \\
& \cdot\left(\gamma W_{11}^{(1)}(-1)+W_{11}^{(2)}(0) e^{-i w_{0} \tau_{0}}\right) .
\end{aligned}
$$
that

Setting $\left(\psi_{1}, \psi_{2}\right)=\Psi_{1}(0)-i \Psi_{2}(0)$, by calculating, we get

$$
\begin{aligned}
& g_{20}=\frac{\tau_{0}}{4}\left(c_{11} \psi_{1}+c_{21} \psi_{2}\right), \\
& g_{02}=\frac{\tau_{0}}{4}\left(c_{12} \psi_{1}+c_{22} \psi_{2}\right), \\
& g_{11}=\frac{\tau_{0}}{8}\left(c_{13} \psi_{1}+c_{23} \psi_{2}\right), \\
& g_{21}=\frac{\tau_{0}}{8}\left(\left\langle c_{01}, 1\right\rangle \psi_{1}+\left\langle c_{02}, 1\right\rangle \psi_{2}\right) .
\end{aligned}
$$

Because there are $W_{20}(\theta)$ and $W_{11}(\theta)$ in $g_{21}$ where $\theta \epsilon$ $[-1,0]$, we still need to compute them.

By [4], we know that

$$
\dot{W}=A_{\tau_{0}} W+H(z, \bar{z}),
$$


where

$$
\begin{aligned}
H(z, \bar{z})= & H_{20} \frac{z^{2}}{2}+H_{11} z \bar{z}+H_{02} \frac{\bar{z}^{2}}{2}+\cdots \\
= & X_{0} f^{*}\left(m^{*}(t), 0\right) \\
& -\Phi\left(\Psi,\left\langle X_{0} f^{*}\left(m^{*}(t), \alpha\right), f_{0}\right\rangle\right) \cdot f_{0},
\end{aligned}
$$

for $H_{i j} \in Q$, with $i+j=2$. It follows from (43), (48), and (49) that

$$
\begin{aligned}
\left(A_{\tau_{0}}-2 i w_{0} \tau_{0}\right) W_{20}(\theta) & =-H_{20}(\theta), \\
A_{\tau_{0}} W_{11}(\theta) & =-H_{11}(\theta), \ldots
\end{aligned}
$$

By (49), we have that for $\theta \in[-1,0)$

$$
\begin{aligned}
H(z, \bar{z})= & -\frac{1}{2}\left[g_{20} p_{1}(\theta)+\bar{g}_{02} p_{2}(\theta)\right] z^{2} \cdot f_{0} \\
& -\left[g_{11} p_{1}(\theta)+\bar{g}_{11} p_{2}(\theta)\right] z \bar{z} \cdot f_{0}+\cdots .
\end{aligned}
$$
$[-1,0)$

Comparing the coefficients with (49), we get that for $\theta \epsilon$

$$
\begin{aligned}
& H_{20}(\theta)=-\left[g_{20} p_{1}(\theta)+\bar{g}_{02} p_{2}(\theta)\right] \cdot f_{0}, \\
& H_{11}(\theta)=-\left[g_{11} p_{1}(\theta)+\bar{g}_{11} p_{2}(\theta)\right] \cdot f_{0} .
\end{aligned}
$$

By (50), (52), and the definition of $A_{\tau_{0}}$, we get that

$$
\begin{aligned}
\dot{W}_{20}(\theta)= & 2 i w_{0} \tau_{0} W_{20}(\theta)+\left[g_{20} p_{1}(\theta)+\bar{g}_{02} p_{2}(\theta)\right] \\
& \cdot f_{0} .
\end{aligned}
$$

$$
E_{1}=\frac{1}{4}\left(\begin{array}{c}
2 i w_{0} \tau_{0}+a_{1}-b_{11} w_{11} g_{1}^{\prime}(0) \\
-b_{21} w_{21} g_{1}^{\prime}(0) e^{-2 i w_{0} \tau_{0}}
\end{array}\right.
$$

In a similar way, substituting (56) and (60) into (58), we obtain that

$$
\begin{aligned}
& E_{2} \\
& =\frac{1}{8}\left(\begin{array}{ll}
a_{1}-b_{11} w_{11} g_{1}^{\prime}(0) & -b_{12} w_{12} g_{2}^{\prime}(0) \\
a_{2}-b_{22} w_{22} g_{2}^{\prime}(0) & -b_{21} w_{21} g_{1}^{\prime}(0)
\end{array}\right)^{-1}\left(\begin{array}{l}
c_{13} \\
c_{23}
\end{array}\right) .
\end{aligned}
$$

Therefore, we can compute the following values:

$$
c_{1}(0)=\frac{i}{2 w_{0}}\left(g_{11} g_{20}-2\left|g_{11}\right|^{2}-\frac{\left|g_{02}\right|^{2}}{3}\right)+\frac{g_{21}}{2},
$$

Noticing that $p_{1}(\theta)=p_{1}(0) e^{i w_{0} \tau_{0} \theta}$, hence,

$$
\begin{aligned}
& W_{20}(\theta) \\
& =\left[\frac{i g_{20}}{w_{0} \tau_{0}} p_{1}(\theta) e^{i w_{0} \tau_{0} \theta}+\frac{i \bar{g}_{02}}{3 w_{0} \tau_{0}} p_{2}(\theta) e^{-i w_{0} \tau_{0} \theta}\right] \cdot f_{0} \\
& \quad+E_{1} e^{2 i w_{0} \tau_{0} \theta},
\end{aligned}
$$

where $E_{1}=\left(E_{1}^{(1)}, E_{1}^{(2)}\right) \in R^{2}$ which is a constant vector.

In a similar way, by (50) and (53), we have that

$$
\begin{aligned}
& W_{11}(\theta) \\
& =\left[-\frac{i g_{11}}{w_{0} \tau_{0}} p_{1}(0) e^{i w_{0} \tau_{0} \theta}+\frac{i \bar{g}_{11}}{w_{0} \tau_{0}} p_{2}(0) e^{-i w_{0} \tau_{0} \theta}\right] \cdot f_{0} \\
& \quad+E_{2},
\end{aligned}
$$

where $E_{2}=\left(E_{2}^{(1)}, E_{2}^{(2)}\right) \in R^{2}$ which is also a constant vector.

In what follows, we seek appropriate $E_{1}$ and $E_{2}$. From the definition of $A_{\tau_{0}}$ and (50), we can obtain that

$$
\begin{aligned}
& 2 i w_{0} \tau_{0} W_{20}(0)-\wp \Delta W_{20}(0)-\mathbb{Z}\left(E^{*}\right) W_{20}(\theta) \\
& \quad=H_{20}(0) \\
& -\wp \Delta W_{11}(0)-\mathbb{Z}\left(E^{*}\right) W_{11}(\theta)=H_{11}(0),
\end{aligned}
$$

where

$$
\begin{aligned}
& H_{20}(0)=\frac{\tau_{0}}{4}\left(\begin{array}{l}
c_{11} \\
c_{21}
\end{array}\right)-\left[g_{20} p_{1}(0)+\bar{g}_{02} p_{2}(0)\right] \cdot f_{0}, \\
& H_{11}(0)=\frac{\tau_{0}}{8}\left(\begin{array}{l}
c_{13} \\
c_{23}
\end{array}\right)-\left[g_{11} p_{1}(0)+\bar{g}_{11} p_{2}(0)\right] \cdot f_{0} .
\end{aligned}
$$

Substituting (55) and (59) into (57), we can obtain that

$\left.\begin{array}{c}-b_{12} w_{12} g_{2}^{\prime}(0) e^{-2 i w_{0} \tau_{0}} \\ 2 i w_{0} \tau_{0}+a_{2}-b_{22} w_{22} g_{2}^{\prime}(0)\end{array}\right)^{-1}\left(\begin{array}{l}c_{11} \\ c_{21}\end{array}\right)$.

$$
\begin{aligned}
& \mu_{2}=-\frac{\operatorname{Re}\left\{c_{1}(0)\right\}}{\operatorname{Re}\left\{\lambda^{\prime}\left(\tau_{0}\right)\right\}}, \\
& \beta_{2}=2 \operatorname{Re}\left\{c_{1}(0)\right\}, \\
& T_{2}=-\frac{\operatorname{Im}\left\{c_{1}(0)\right\}+\mu_{2} \operatorname{Im}\left\{\lambda^{\prime}\left(\tau_{0}\right)\right\}}{w_{0}},
\end{aligned}
$$

which determine the quantities of bifurcating periodic solutions in the center manifold at the critical value $\tau_{0}$; that is, $\mu_{2}$ determines the direction of Hopf bifurcation: the Hopf bifurcation is supercritical (subcritical) if $\mu_{2}>0\left(\mu_{2}<0\right)$ and the bifurcating periodic solutions exist for $\tau>\tau_{0}(\tau<$ 
$\left.\tau_{0}\right) ; \beta_{2}$ determines the stability of the bifurcating periodic solutions: if $\beta_{2}<0\left(\beta_{2}>0\right)$, the bifurcating periodic solutions are stable (unstable); and $T_{2}$ determines the period of the bifurcating periodic solutions: the period increases (decrease) if $T_{2}>0\left(T_{2}<0\right)[8-11]$.

\section{Numerical Simulations}

In this section, in order to illustrate the results above, we will give two examples.

Example 1. In system (2), we choose that $D_{1}=D_{2}=1, a_{1}=$ $b_{11}=0.4, w_{11}=0.6, a_{2}=0.3, b_{22}=w_{22}=0.5, b_{12}=0.3$, $b_{21}=0.6, w_{12}=2.4, w_{21}=3.6, g_{1}(x)=-0.1 \tan (x)$, and $g_{2}(x)=\arctan (x)$; then,

$$
\begin{aligned}
\frac{\partial u}{\partial t}= & \Delta u-0.4 u(t, x)-0.04 \tan (0.6 u(t, x)) \\
& +0.3 \arctan (2.4 v(t-\tau, x)) \\
\frac{\partial v}{\partial t}= & \Delta v-0.3 v(t, x)-0.06 \tan (3.6 u(t-\tau, x)) \\
& +0.5 \arctan (0.5 v(t, x))
\end{aligned}
$$

in which

$$
\begin{aligned}
& 0<x<1, \\
& t>0
\end{aligned}
$$

with initial and Neumann boundary conditions

$$
\begin{aligned}
\frac{\partial u}{\partial l} & =\frac{\partial v}{\partial l}=0, \quad t \geq 0, x=0,1, \\
u(t, x) & =0.5\left(1+\frac{t}{\pi}\right) \sin (\pi x), \\
v(t, x) & =\left(1+\frac{t}{\pi}\right) \sin (\pi x), \\
& (t, x) \in[-\tau, 0] \times[0,1] .
\end{aligned}
$$

What should be remarked is that we choose the parameter values stochastically under the condition $q_{2}<0$ in order to ensure the existence of Hopf bifurcation at $E^{*}$ when $\tau=\tau_{0}$.

So, $\tau_{0}=1.9371$ and $w_{0}=0.2939$. Then, we can know on the basis of Theorem 1 that the trivial uniform steady state $E^{*}=(0,0)$ is asymptotically stable when $0 \leq \tau<\tau_{0}$. When $\tau>\tau_{0}$, the steady state is unstable and a Hopf bifurcation is arising from the steady state. The numerical simulations in Figures 1 and 2 illustrate the facts.

When $\tau=\tau_{0}$, we get that $c_{1}(0)=-0.0001+0.0022 i$; then, we can acquire that $\mu_{2}>0$ and $\beta_{2}<0$. Hence, when $\tau$ passes through $\tau_{0}$ to the right $\left(\tau>\tau_{0}\right.$ ), the bifurcation turns up, and the corresponding periodic orbits are orbitally asymptotically stable.

Example 2. In system (2), we choose that $D_{1}=D_{2}=0.01$, $b_{21}=0.9, a_{2}=0.2, b_{12}=0.3, b_{11}=b_{22}=w_{22}=0.5, a_{1}=$ $w_{11}=0.6, w_{12}=2.5, w_{21}=3.6, g_{1}(x)=-0.1 \tan (x)$, and $g_{2}(x)=\arctan (x)$; then,

$$
\begin{aligned}
\frac{\partial u}{\partial t}= & 0.01 \Delta u-0.6 u(t, x)-0.05 \tan (0.6 u(t, x)) \\
& +0.3 \arctan (2.5 v(t-\tau, x)) \\
\frac{\partial v}{\partial t}= & 0.01 \Delta v-0.2 v(t, x)-0.09 \tan (3.6 u(t-\tau, x)) \\
& +0.5 \arctan (0.5 v(t, x))
\end{aligned}
$$

in which

$$
\begin{aligned}
& 0<x<1, \\
& t>0
\end{aligned}
$$

with initial and Dirichlet boundary conditions

$$
\begin{aligned}
& u(t, 0)=u(t, 1)=v(t, 0)=v(t, 1)=0, \quad t \geq 0, \\
& u(t, x)=0.5\left(1+\frac{t}{\pi}\right) \sin (\pi x), \\
& v(t, x)=\left(1+\frac{t}{\pi}\right) \sin (\pi x),
\end{aligned}
$$

$$
(t, x) \in[-\tau, 0] \times[0,1] .
$$

The similar Hopf bifurcation phenomenon is illustrated by the numerical simulations in Figures 3 and 4 .

\section{Discussion and Research Perspective}

This section is devoted to a summary of discussion and research perspective for the generalized reaction-diffusion neural network model. The model is based on the assumption that the signal transmission is of a digital (McCulloch-Pitts) nature; the model then described a combination of analog and digital signal processing in the network [12]. Depending on the modeling approaches, neural networks can be modeled either as a static neural network model or as a local field neural network model. In order to let the problem be more general in many aspects, we build a generalized reactiondiffusion neural network model which includes reactiondiffusion local field neural networks and reaction-diffusion static neural networks. For a delayed neural network, an important issue is the dynamical behaviors of the network [13]. Thus, there has been a large body of work discussing the stability and bifurcation in delayed neural network models. By analyzing the characteristic equation, we discussed the local stability of the trivial uniform of system (2) [14]. It was shown that when the delay $\tau$ varies, the trivial uniform steady state exchanges its stability and Hopf bifurcations occur. Numerical simulations illustrated the occurrence of the bifurcate periodic solutions when the delay $\tau$ passes the critical value $\tau_{0}$.

A research perspective includes the problem of determining the bifurcating periodic solutions and the stability and directions of the Hopf bifurcation using the normal 


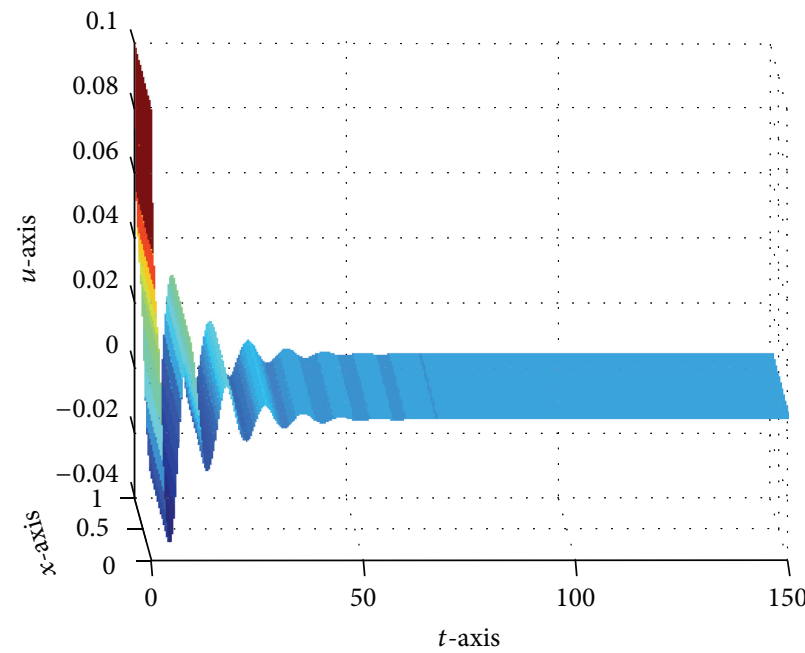

(a)

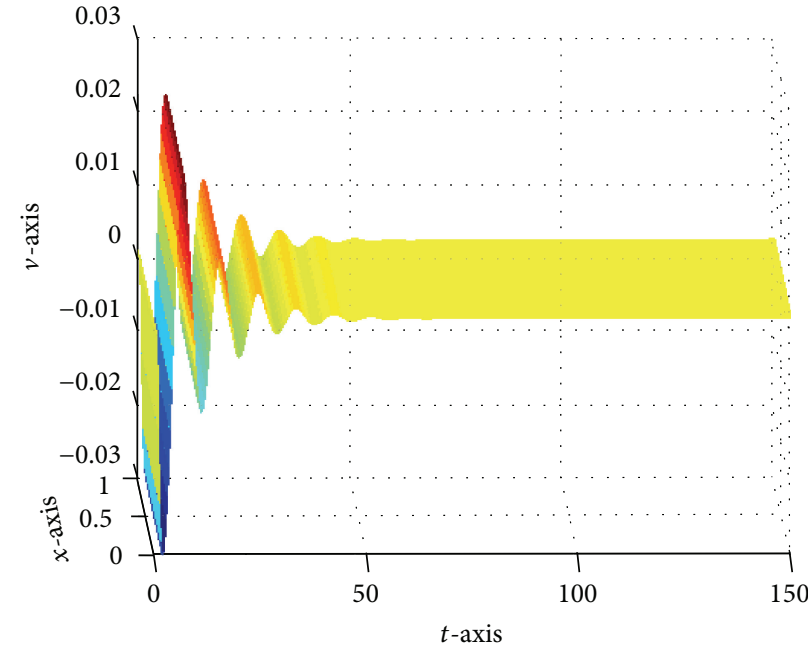

(b)

FIGURE 1: The temporal solution found by numerical integration of systems (64) and (66) with $\tau=1.85$ : (a) $u(t, x)$ and (b) $v(t, x)$.

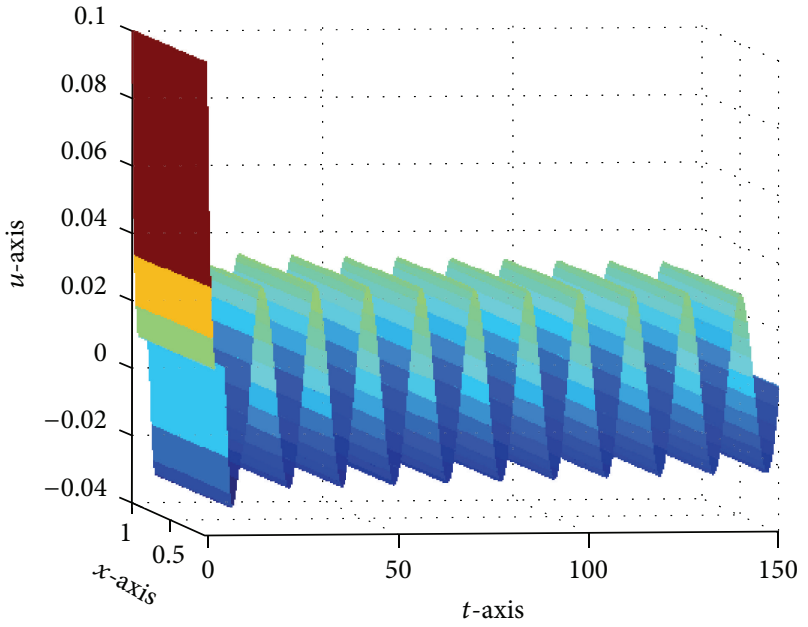

(a)

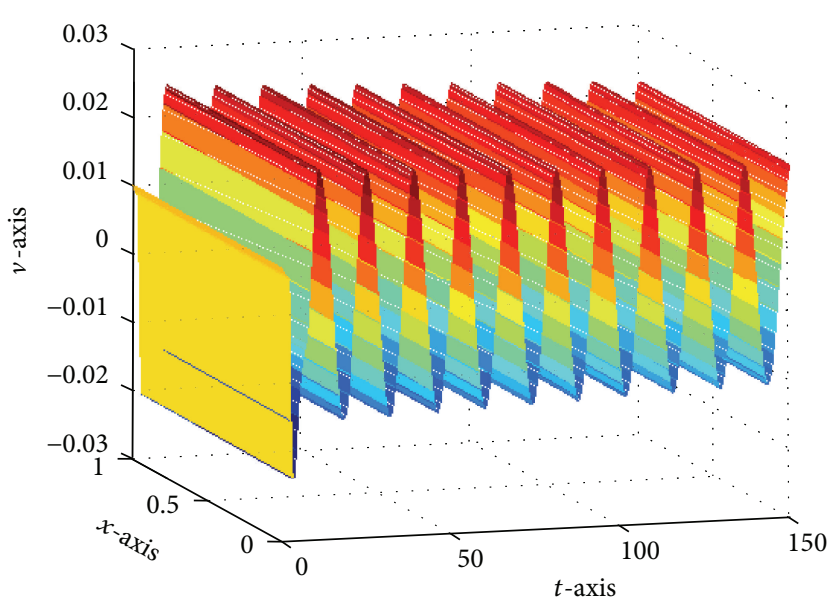

(b)

FigURE 2: The temporal solution found by numerical integration of systems (64) and (66) with $\tau=4.25:(\mathrm{a}) u(t, x)$ and (b) $v(t, x)$.

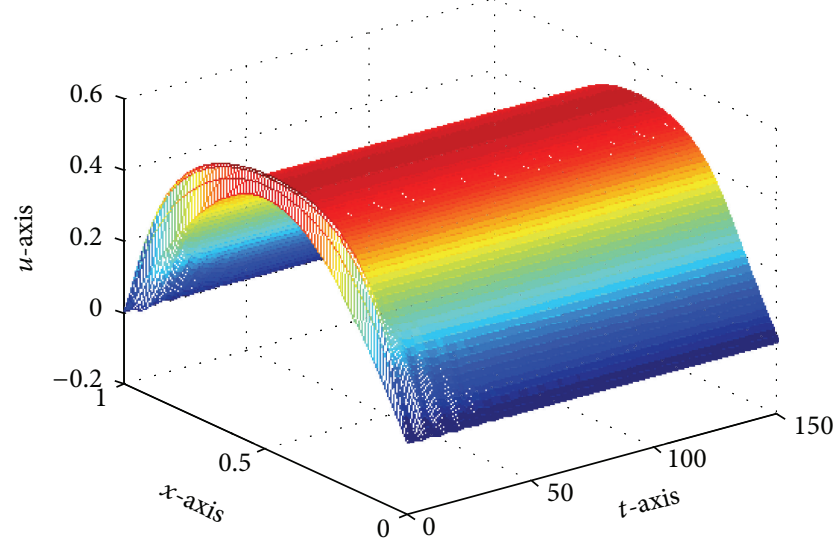

(a)

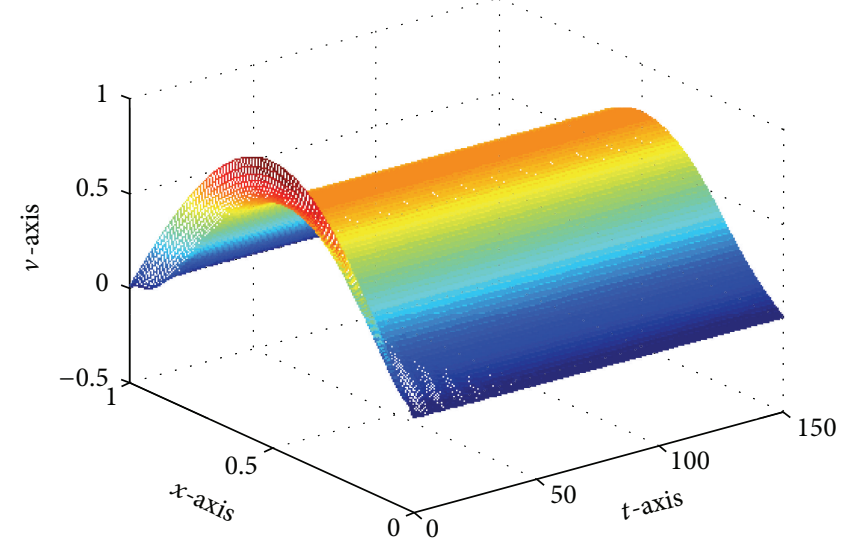

(b)

FIgURE 3: The temporal solution found by numerical integration of systems (67) and (69) with $\tau=1.25:(\mathrm{a}) u(t, x)$ and (b) $v(t, x)$. 


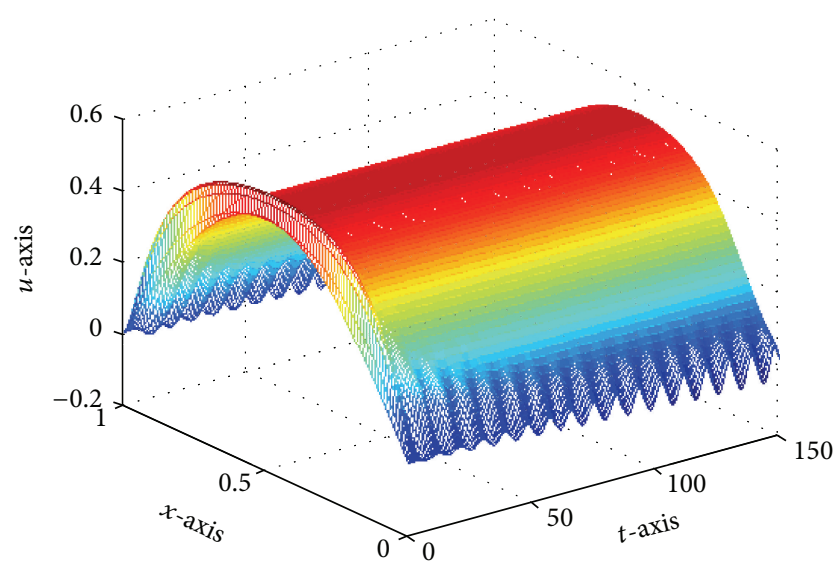

(a)

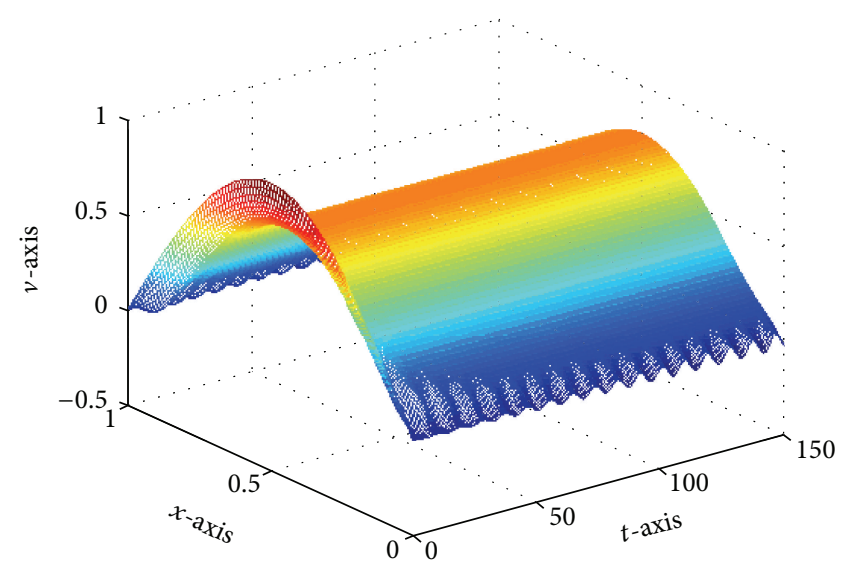

(b)

FIGURE 4: The temporal solution found by numerical integration of systems (67) and (69) with $\tau=2.05:(\mathrm{a}) u(t, x)$ and (b) $v(t, x)$.

form theory and the center manifold reaction. A challenging perspective is the comparison of the generalized model introduced in the present paper with the experimentally measurable quantities. Indeed, the mathematical models should reproduce both qualitatively and quantitatively empirical data (see [4]).

\section{Conflict of Interests}

The authors declare that there is no conflict of interests regarding the publication of this paper.

\section{Acknowledgments}

This work was supported by the National Natural Science Foundation of China (no. 61305076) and the Scientific Research Foundation for the Returned Overseas Chinese Scholars, State Education Ministry.

\section{References}

[1] C. Bianca, M. Ferrara, and L. Guerrini, "The time delays' effects on the qualitative behavior of an economic growth model," Abstract and Applied Analysis, vol. 2013, Article ID 901014, 10 pages, 2013.

[2] L. V. Ballestra, L. Guerrini, and G. Pacelli, "Stability switches and bifurcation analysis of a time delay model for the diffusion of a new technology," International Journal of Bifurcation \& Chaos, vol. 24, no. 9, Article ID 1450113, 2014.

[3] Q. Gan and R. Xu, "Stability and Hopf bifurcation of a delayed reaction-diffusion neural network," Mathematical Methods in the Applied Sciences, vol. 34, no. 12, pp. 1450-1459, 2011.

[4] C. Bianca and L. Guerrini, "On the Dalgaard-Strulik model with logistic population growth rate and delayed-carrying capacity," Acta Applicandae Mathematicae, vol. 128, pp. 39-48, 2013.

[5] O. G. Jepps, C. Bianca, and L. Rondoni, "Onset of diffusive behavior in confined transport systems," Chaos, vol. 18, no. 1, Article ID 013127, 2008.

[6] L. Gori, L. Guerrini, and M. Sodini, "Equilibrium and disequilibrium dynamics in cobweb models with time delays,"
International Journal of Bifurcation and Chaos, vol. 25, no. 6, Article ID 1550088, 2015.

[7] C. Bianca, L. Guerrini, and A. Lemarchand, "Existence of solutions of a partial integrodifferential equation with thermostat and time delay," Abstract and Applied Analysis, vol. 2014, Article ID 463409, 7 pages, 2014.

[8] Y. Kuang, "Delay differential equations with applications in population dynamics," Discrete \& Continuous Dynamical Systems, vol. 33, no. 4, pp. 1633-1644, 2013.

[9] C. Huang, Y. He, L. Huang, and Y. Zhaohui, "Hopf bifurcation analysis of two neurons with three delays," Nonlinear Analysis: Real World Applications, vol. 8, no. 3, pp. 903-921, 2007.

[10] C. Huang, L. Huang, J. Feng, M. Nai, and Y. He, "Hopf bifurcation analysis for a two-neuron network with four delays," Chaos, Solitons \& Fractals, vol. 34, no. 3, pp. 795-812, 2007.

[11] Y. Song, M. Han, and J. Wei, "Stability and Hopf bifurcation analysis on a simplified BAM neural network with delays," Physica D: Nonlinear Phenomena, vol. 200, no. 3-4, pp. 185-204, 2005.

[12] X.-P. Yan, "Hopf bifurcation and stability for a delayed trineuron network model," Journal of Computational and Applied Mathematics, vol. 196, no. 2, pp. 579-595, 2006.

[13] H. Zhao and L. Wang, "Hopf bifurcation in Cohen-Grossberg neural network with distributed delays," Nonlinear Analysis: Real World Applications, vol. 8, no. 1, pp. 73-89, 2007.

[14] B. Zheng, Y. Zhang, and C. Zhang, "Stability and bifurcation of a discrete BAM neural network model with delays," Chaos, Solitons \& Fractals, vol. 36, no. 3, pp. 612-616, 2008. 


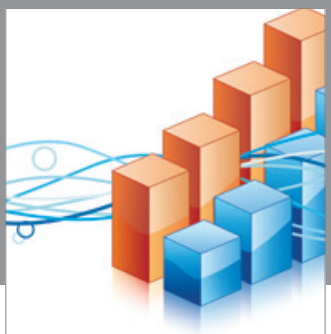

Advances in

Operations Research

vatem alat4

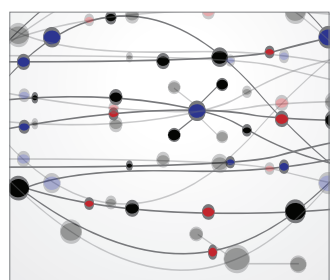

\section{The Scientific} World Journal
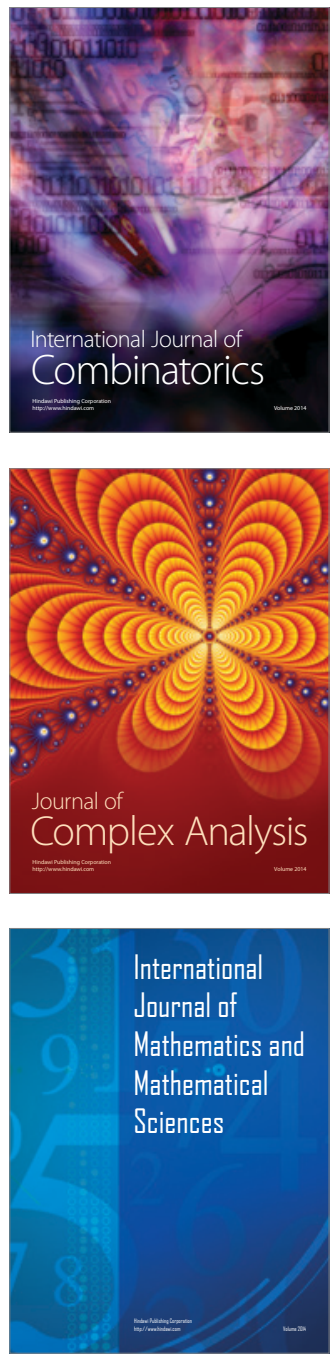
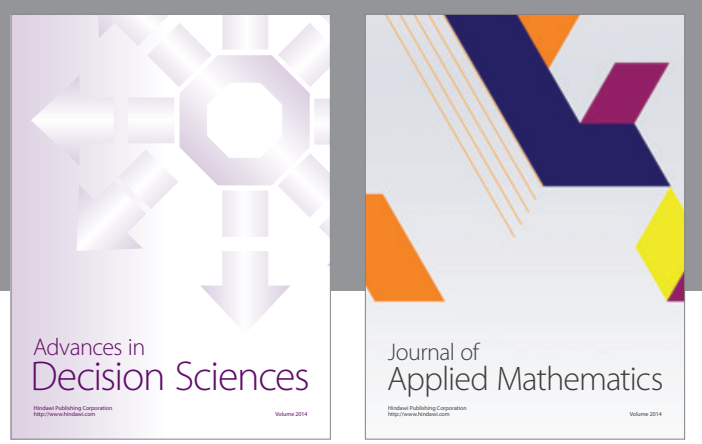

Algebra

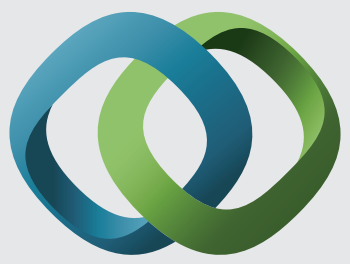

\section{Hindawi}

Submit your manuscripts at

http://www.hindawi.com
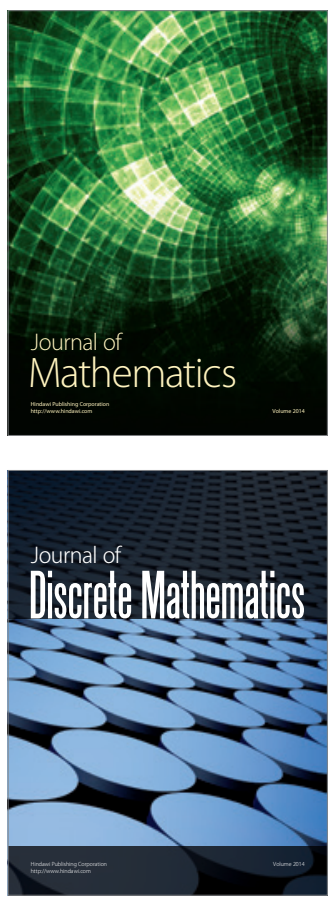

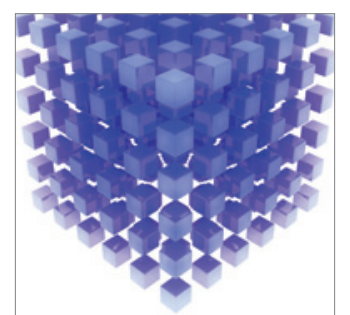

Mathematical Problems in Engineering
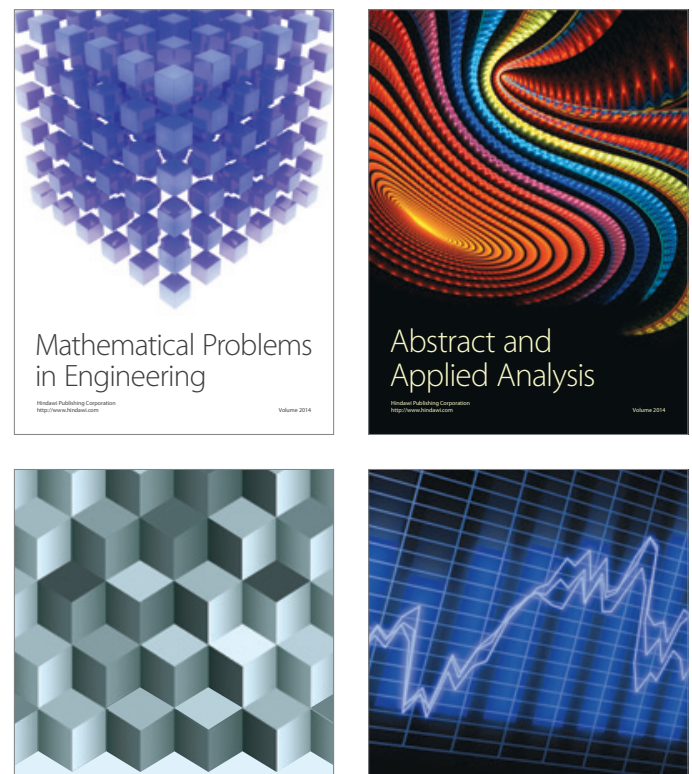

Journal of

Function Spaces

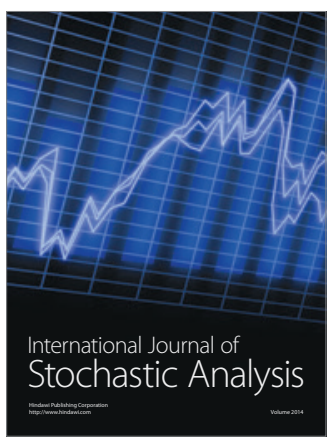

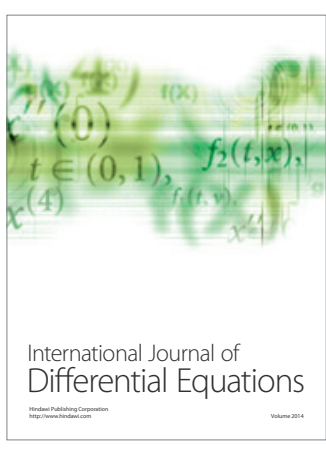
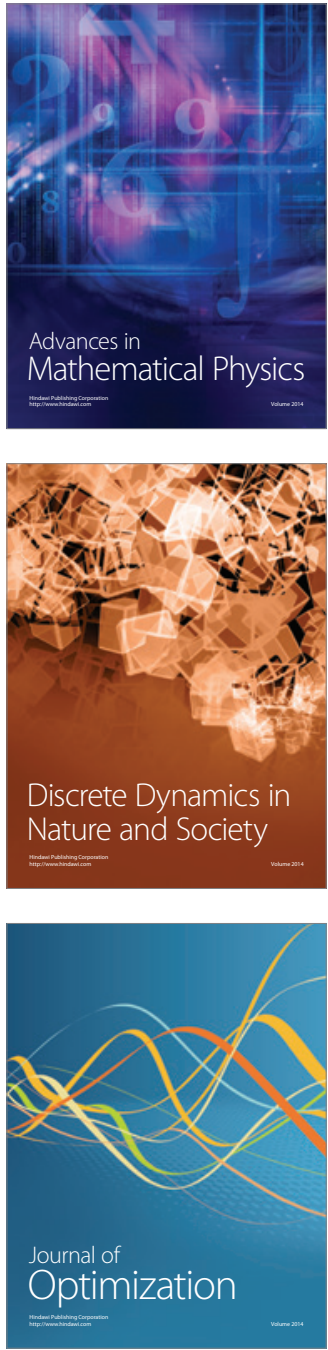